\title{
The role of semantic knowledge in retrieval from episodic long-term memories: Implications for a model of retrieval
}

\author{
JANE PERLMUTTER, JACKEE HARSIP, and JEROME L. MYERS \\ University of Massachusetts, Amherst, Massachusetts 01002
}

\begin{abstract}
Two experiments were conducted, using a paired-associate recall reaction time paradigm, to assess the role semantic knowledge plays in retrieval of long-term episodic memories. In Experiment I, word frequency and meaningfulness were manipulated; high-frequency stimuli led to significantly longer reaction times, while meaningfulness had no significant effect. In Experiment II, the role of the distribution of preexperimental associates of the stimulus was further investigated by comparing retrieval time when the stimulus had either a high- or low-strength primary associate; the former resulted in longer reaction times. The implications of these results for a model of retrieval were discussed. Exhaustive search models were found inadequate, but viable parallel and serial self-terminating models were developed. In both of these models, retrieval time is a function of strength of the correct associate, relative to total strength of a restricted set of semantic associates of the stimulus.
\end{abstract}

A major current concern of cognitive psychologists is the specification of those processes involved in retriev. ing information from long-term memory (LTM). In the paradigm we have used to investigate this issue (Perlmutter, Sorce, \& Myers, 1976), subjects were required to memorize lists of paired associates ( 3 to 24 PAs) and then, on each of several hundred randomly sequenced trials, time to vocalize the correct response to a visually presented stimulus was measured. Such recall times increased as a function of list length and decreased with probe probability and probe recency. Furthermore, both the slope and intercept of the response time list length function decreased with practice within an experimental session. The fact that these functions did not vary across sessions (subjects were assigned new lists for each session) demonstrated that such practice effects were item specific. Finally, responses which were preexperimentally associated to the stimulus word (e.g., DOG-CAT) were recalled more quickly than nonassociates (e.g., DOG-CHAIR), even after several days of practice with the same list (Perlmutter, Sorce, Harsip, \& Myers, Note 3). This associative strength effect decreased as a function of practice and probability and recency of the probe.

In attempting to specify a retrieval model, we concluded that scanning models (e.g., Sternberg, 1969; Theios, 1973), which emphasize the role short-term memory (STM) plays in retrieval, cannot fully encom-

This research was supported by a National Science Foundation Grant, NSF GU-4041, by a National Institute of Mental Health Grant, MH 25434-01, and a grant for free computer time from the University Computing Center, University of Massachusetts at Amherst. Requests for reprints should be sent to Jerome L. Myers, Department of Psychology, University of Massachusetts, Amherst, Massachusetts 01002. pass these results. The effects of associative strength and of item-specific practice were both present even when the list was within the capacity of STM. We concluded that LTM is a network of concepts and that, within the context of such a network, the relations between the concepts have a strength value which increases when the relevant item is probed and decreases when other items are probed. Response time (RT) is then assumed to decrease as strength of the probed association increases. A stochastic model derived from these assumptions predicted the results cited above and, in fact, provided an excellent fit to the fine grain of the data. The model could also account for errors in recall; for example, the strength might be so low that the correct response was not retrieved prior to some deadline.

This model implies direct access to the response from the stimulus node. An alternative view would be that the number and strengths of other associations to the stimulus also influence retrieval. This second hypothesis would be more consistent with various formulations of interference theory (cf. Postman \& Underwood, 1973) and with several recently proposed models in which retrieval is based on a ratio-of-strengths rule (Rundus, 1973; Shiffrin, 1970). In this case, the higher the proportion of strength of the correct associate, the higher the likelihood that it will be retrieved correctly and rapidly. One purpose of the experiments reported here was to distinguish between the "direct access to response" and the "competing associates" hypotheses.

If natural language associates of the stimulus are shown to compete with the leamed response, it would argue that episodic memories are strongly dependent upon their semantic referents (see Reder, Anderson, \& 


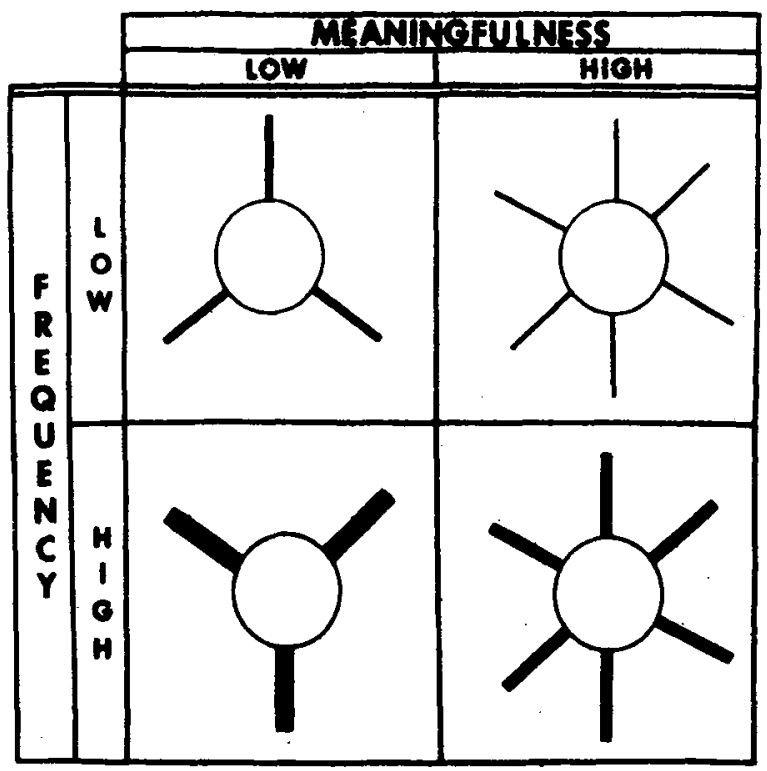

Figure 1. Schematic representation of stimuli as a function of $f$ and $m$.

Bjork, 1974; Watkins \& Tulving, 1975). Furthermore, as the strength of such competing associates increases, so should RT to retrieve an experimentally paired associate. One approach to manipulating the strengths of these associations is suggested by the work of Saltz (1967) and Modigliani \& Saltz (1969), who found that the rate of PA learning decreased as a function of frequency of English language usage $(f)$ of the stimulus. Saltz (1967) has identified $f$ with the totality of strength of associations of the stimulus word, and has argued that the observed $f$ effect reflects the degree of competition from such associates. While we recognize that $\mathrm{f}$ is also an index of other properties of words (cf. Landauer \& Streeter, 1973), such properties consistently result in swifter encoding of higher $f$ words and should, therefore, yield shorter recall times in our PA paradigm. To the extent that recall time increases with stimulus $f$, both Saltz's identification of $f$ with total strength and the hypothesis that the strength of semantic associations influences episodic retrieval will receive support.

Saltz (1967) also found that learning rate increased with increases in stimulus meaningfulness ( $\mathrm{m}$; Paivio, Yuille, \& Madigan, 1968). Under the procedures by which $\mathrm{m}$ norms are obtained, it is reasonable to view $\mathrm{m}$ as an index of number of associations to the stimulus. At any given level of $f, m$ provides one way of manipulating the distribution of competing strengths of associates. Given our identification of total strength with $f$, it follows that the average strengths of associations to a stimulus will vary inversely with $m$ when $f$ is fixed. Figure 1 presents a somewhat idealized representation of this view of $f$ and $m$. If $R T$ decreases as $m$ increases, as the analogy to Saltz's work would suggest, it would indicate that the proportion of total strength attached to the memorized associate does not completely determine the retrieval process. Rather, some mechanism responsive to the distribution of strengths among associations would need to be incorporated into the search model.

In summary, the experiments reported in the current paper investigated the retrieval of well-memorized associates. In particular, we were concerned with the extent to which preexperimental associations influence retrieval of the memorized associate. Having found that such associations do play a role, we have attempted to characterize the processes involved. For example, is the search serial or parallel, exhaustive or selfterminating, with or without replacement? The experiments to be presented will allow us to reject some models and will implicate a plausible subset for future research. As we have indicated, we will manipulate stimulus $f$, viewing it as an index of competing strength. In addition, manipulations of stimulus $m$ and strength of the stimulus' primary associate will provide two approaches to varying the distribution of strengths at fixed $f$.

\section{EXPERIMENT I}

\section{Method}

Design and subjects. Twenty-four students at the University of Massachusetts served as subjects. All subjects learned the same 12-item list. One-fourth of the stimulus words were from each cell of a 2 by 2 design, with word $f$ of use in English being high or low and word $\mathrm{m}$ being high or low.

Materials. The following PAs were used in Experiment I: high $f$, low m-FACT-FRIEND, FORM-CHANGE, MONTHSEAT; high $f$, high $m$-CHAIR-HOPE, STORM-GOLD, QUEENMIND; low f, low m-BARD-ROCK, GIST COST, KEG-CHIEF; low $f$, high $m$-SUDS-AIR, WHALE-HALL, YACHT-DRESS. All words were one-syllable nouns, between three and six letters long. All response words were high-f (AA in the Thorndike-Lorge norms) and intermediate-m words (mean $\mathrm{m}=$ 6.03 , range between 5.52 and 6.40 in the Paivio et al. norms). Half of the stimuli were high-f words (AA in the ThorndikeLorge norms) and half were low-f words (mean ThorndikeLorge frequency $=4.18$, range between 1 and 8 ). Half of each group of stimuli were high $\mathrm{m}$ (mean $\mathrm{m}=7.25$, range between 7.20 and 7.36 in the Paivio et al. norms) and half were low $\mathrm{m}$ (mean $\mathrm{m}=4.40$, range between 3.33 and 4.80 in the Paivio et al. norms). Both imaginability and concreteness (Paivio et al., 1968) were completely confounded with $m$ of the stimuli, but were independent of $f$. Stimuli and responses were randomly paired, and none of the responses were associates of their stimulus (Palermo \& Jenkins, 1964).

Procedure and apparatus. Each subject participated in a 1-h experimental session which consisted of 12 blocks of 48 trials. A PDP/8 computer controlled the sequencing and timing of stimuli, and recorded stimuli, responses, and RT for all trials. Stimulus members of the PA were randomly presented (with the restriction that all PAs were presented equally often in each block) on a video display monitor, and subjects were required to vocalize a response as quickly as possible. The subject's response triggered a voice key which caused the removal of the stimulus from the screen and the appearance of the correct response word, as well as the subject's RT. The subject then pulled one of two levers to indicate whether or not his response was correct. The RT was measured from the onset of the 
Table 1

Mean RT (Milliseconds): Experiment I

\begin{tabular}{cccc}
\hline Frequency & Low & $\begin{array}{c}\text { Meaningfulness } \\
\text { High }\end{array}$ & Mean \\
\hline Low & 769 & 717 & 743 \\
High & 916 & 873 & 894 \\
Mean & 843 & 795 & 819 \\
\hline
\end{tabular}

stimulus until the triggering of the voice key. The time between consecutive probes was $3.5 \mathrm{sec}$, and the onset of a probe was preceded by a .5 -sec warning tone.

The first two trial blocks were READ blocks, in which the subject simply read the stimulus word from the screen. This provided baseline times, assumed to reflect the time spent in stimulus encoding and response execution stages. In addition, it gave the subject an opportunity to learn the PAs while controlling rehearsal, and served to familiarize the subject with the apparatus and reduce item-independent practice effects in the RECALL data. The final 10 trial blocks were RECALL blocks, in which the subject said the response word that he had learned to associate to the word on the video display.

\section{Results}

Mean RT of correct responses for individual PAs is the dependent variable of interest in Experiment $\mathrm{I}$, and thus the quasi-F statistic (Myers, 1972) will be reported where appropriate. Overall error rates in recall were: high $\mathrm{f}$, low $\mathrm{m}-14.7 \%$; high $\mathrm{f}$, high $\mathrm{m}-10.4 \%$; low $\mathrm{f}$, low $\mathrm{m}-6.4 \%$; low $\mathrm{f}$, high $\mathrm{m}-2.4 \%$. An indeterminate proportion of these errors were caused by occasional failures of the voice key to register a response. As we shall see shortly, mean recall RT fell in the same order as error rates. Thus, the results cannot be interpreted in terms of a speed-accuracy tradeoff.

READ task. While there was significant variability in READ time for different words in this experiment $\left[F(8,184)=3.0, p<.05, M_{e}=30,920\right]$, it can neither be attributed to $\mathrm{f}\left[q\right.$ quasi-F $(1,7)<1.0, \mathrm{p}>.05, \mathrm{MS}_{\mathrm{e}}=$ 85,550 ], to $\mathrm{m}$ [quasi- $\mathrm{F}(1,7)<1.0, \mathrm{MS}_{\mathrm{e}}=85,961$ ], nor to their interactions [quasi- $\mathrm{F}(1,7)<1.0, \mathrm{MS}_{\mathrm{e}}=$ $89,908]$. The RT was faster for the second READ block $\left[\mathrm{F}(1,23)=4.7, \mathrm{p}<.05, \mathrm{MS}_{\mathrm{e}}=156,033\right]$, presumably reflecting the subjects' initial unfamiliarity with the apparatus.

RECALL task. Table 1 presents mean RT for RECALL blocks. As in the READ task, there was significant variability among words in the same cell of this experiment $\left[\mathrm{F}(8,184)=11.4, \mathrm{p}<.01, \mathrm{MS}_{\mathrm{e}}=162,422\right]$. However, in the RECALL task, average RT was $150 \mathrm{msec}$ slower to high- than low-f stimuli [quasi$\mathrm{F}(1,0)=10.3, \mathrm{p}<.01, \mathrm{MS}_{\mathrm{e}}=191,9571$. Stimulus $\mathrm{m}$, on the other hand, did not significantly affect RT [quasi- $\left.\mathrm{F}(1,8)<1.0, \mathrm{MS}_{\mathrm{e}}=1,856,652\right]$. Furthermore, the interaction of Stimulus $f$ by $m$ was not significant [quasi- $\mathrm{F}(1,8)<1.0, \mathrm{MS}_{\mathrm{e}}=1,894,231$ ]. In this experiment, there was a significant practice effect $[F(9,173)=$ $\left.2.2, \mathrm{p}<.05, \mathrm{MS}_{\mathrm{e}}=248,751\right]$; however, this effect was a function of neither stimulus $f$ [quasi- $F(9,59)=$
$1.1, \mathrm{p}>.05, \mathrm{MS}_{\mathrm{e}}=81,981$ ] nor stimulus $\mathrm{m}$ [quasi$\mathrm{F}\left(9,53 j<1.0, \mathrm{p}>.05, \mathrm{MS}_{\mathrm{e}}=76,431\right]$.

A final measure which we have found useful in drawing inferences about retrieval processes is the slope of the lag function, where lag is defined as the number of intervening probes between successive trials with the same PA. The lag functions for the four types of stimuli are presented in Figure 2. As can be seen, these functions are not linear; however, assessing the direction and magnitude of their linear components will indicate the nature and relative size of the effect of lag on RT. This was accomplished by calculating the slope of the best-fitting linear RT-lag function for each subject, for each stimulus type, and for each block, and submitting these slopes to an analysis of variance. These functions were increasing with a mean slope of $1.69[F(1,23)=$ $\left.28.4, \mathrm{p}<.01, \mathrm{MS}_{\mathrm{e}}=97\right]$. In this experiment, more frequent and less meaningful stimuli had steeper lag functions $\left[F(1,23)=11.2, \mathrm{p}<.01, \mathrm{MS}_{\mathrm{e}}=69\right.$ and $\mathrm{F}(1,23)=12.2, \mathrm{p}<.01, \mathrm{MS}_{\mathrm{e}}=45$, for the $\mathrm{f}$ and $\mathrm{m}$ effects, respectively]. The interaction effect was also significant $\left[\mathrm{F}(1,23)=4.3, \mathrm{p}<.05, \mathrm{MS}_{\mathrm{e}}=43\right]$.

\section{Discussion}

In Experiment $\mathrm{I}$, neither stimulus $\mathrm{f}$ nor $\mathrm{m}$ affected

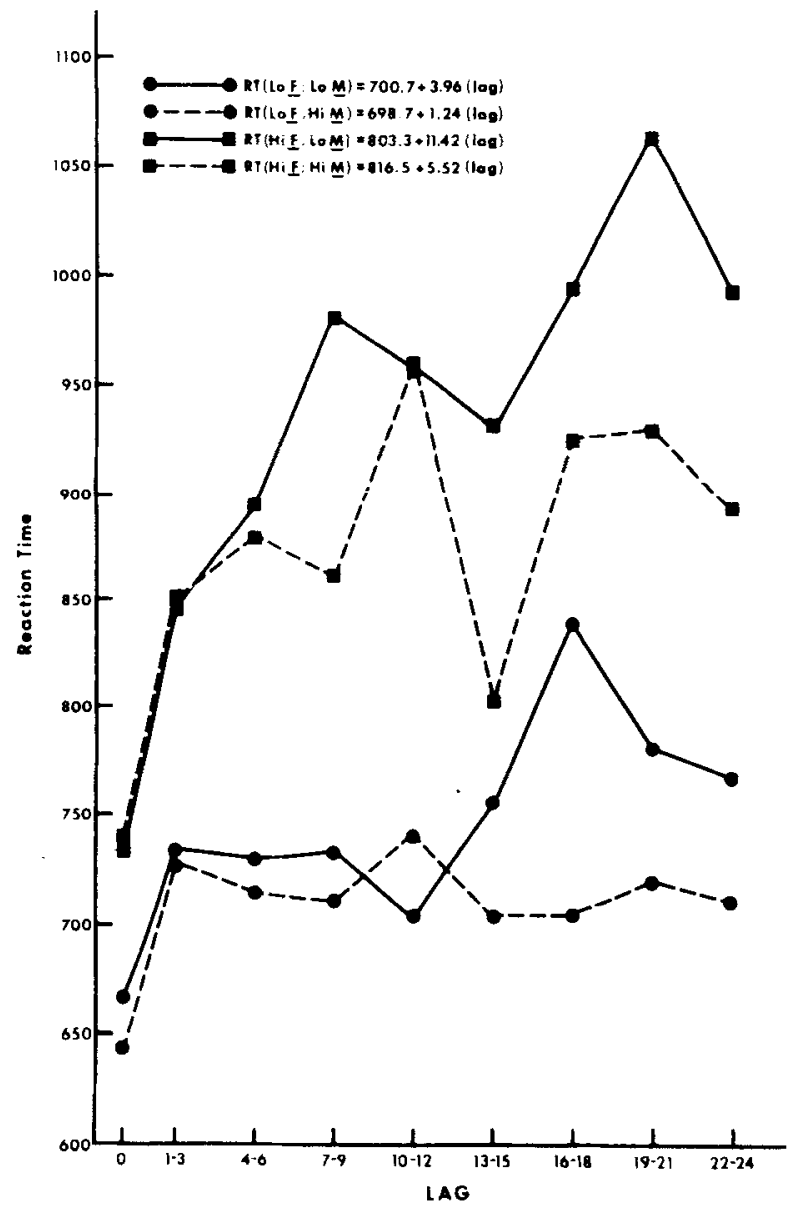

Figure 2. Lag functions: Experiment I. 
READ time. Other research (Forster \& Chambers, 1973), including a preliminary study of our own (Perlmutter, Harsip, \& Myers, Note 2), has generally found that RT is shorter for high-f words; presumably, the fact that such a difference was not obtained in Experiment $I$ is attributable to the relatively high variability of the READ data. The subjects' primary responsibility during these blocks was to familiarize themselves with the apparatus and to learn the list; long and variable RT, and evidence of a practice effect, attest to the fact that subjects were not reading as fast as possible.

The major point of interest in Experiment $I$ was assessment of the independent influences of stimulus $f$ and $m$ upon retrieval. Stimulus $f$ clearly plays an important role in this process; overall RT was longer and slopes of lag functions were steeper for high-f stimuli. Because the sample of items is limited, it is important to note that similar significant effects were observed with other items in a preliminary study (Perlmutter et al., Note 2).

The longer RT for these stimuli is consistent with the higher error rate observed by Saltz (1967) and by Modigliani and Saltz (1969), and with the competitive search argument advanced in the introduction. On the other hand, the status of stimulus $m$ is less clear. While the main effect of $m$ on mean RT is clearly not significant, the 50 -msec difference observed is not trivial in the general context of recall RT experiments, and the effect upon both RT and error rate is in the same direction observed by Saltz (1967). Perhaps more pertinent is the finding that lag functions were significantly steeper for low- than high-m stimuli, suggesting that stimulus $m$ does play some role in processing.

Saltz (1967) and Modigliani and Saltz (1969) interpreted the effects of stimulus $f$ and $m$ in terms of the strength of competing preexperimental associates. They reasoned that increasing $f$, and decreasing $m$, resulted in higher average strength (greater total strength distributed among fewer associations) of such associations to the stimulus. Although the effect of $m$ is equivocal in the present experiment, the actual distribution of strengths may influence retrieval more clearly. An experiment reported by Shapiro (1968) supports this possibility. The RT to retrieve nonprimary associates of equal associative strength to the stimuli was faster if the stimulus had a high-strength primary associate than if it did not. Unfortunately, closer examination of Shapiro's material (Note 4) indicates a slight confounding of this manipulation with stimulus $f$ and S-R associative strength; that is, the direction of RT differences could be predicted solely on the basis of small differences in associative strength (the high primary stimuli were paired with stronger response associates) and stimulus $f$ (the high primary stimuli were less frequently used words). Experiment II will explore this issue further.

\section{EXPERIMENT II}

\section{Method}

Design and subjects. Twelve students at the University of Massachusetts served as subjects. fill students learned the same 12-item list. One half of the stimulus words had high associates as their primary free associates, while the other half of the stimuli had lower strength primary associates. Each subject gave two blocks of READ data, during which time he leamed the PAs followed by nine blocks of RECALL data; the final (12th) block of the experiment was also a READ block. All blocks consisted of 48 trials.

Materials. The following PAs were used in Experiment II: low primary-BISCUIT-SEAT, LEATHER-HALL, MESSAGEAIR, OVEN-CHIEF, OYSTER-DRESS, SIDEWALK-FRIEND; high primary-AUTHOR-GOLD, CANDLE-MIND, CIRCUSROCK, DRAMA-CHANGE, FOREST-COST, REGION-HOPE. All stimuli were two-syllable nouns, between four and eight letters long. Half of the stimuli had high-strength primary associates (mean $=46.5 \%$ using the continuous association procedure and mean $=48.5 \%$ using the discrete association procedure in the Postman, 1970, norms), while the other half had low-strength primary associates (mean $=17.0 \%$ with the continuous association procedure and mean $=18.3 \%$ with the discrete association procedure). The two groups of stimuli were equated for word $f$ (mean $L$ count $=180$ and 202 for the high and low primary stimuli, respectively; ThorndikeLorge, 1944) and word $m$ (mean $m=9.9$ for both groups in the Postman, 1970, norms).

Responses were all one-syllable nouns from three to six letters long. They were all high-f words (AA in the ThorndikeLorge, 1944, norms) and intermediate in $\mathrm{m}$ (mean $=\mathrm{m}=6.06$ in the Paivio et al. norms, 1968). Stimuli and responses were randomly paired and none of the responses were associates of their stimuli (Palermo \& Jenkins, 1964).

Procedure and apparatus. The procedure and apparatus were the same as in Experiment I, except that the final block was a READ block in this experiment.

\section{Results}

Overall error rates differed only slightly: $.8 \%$ for the high primary condition and $.4 \%$ for the low primary condition. The difference is, as we shall see, in the same direction as the RT effect. Thus, there is again no evidence for a speed-accuracy tradeoff. Rather, the data are again consistent with the notion that accuracy and recall time effects are consequences of the same underlying process.

READ task. Since the READ time during the first two blocks was collected while subjects were acquainting themselves with the list and apparatus, only the last block of READ data (i.e., following the RECALL task) was analyzed. The level of strength of the primary associate had no effect on time to read the stimulus (mean RT $=453$ and $441 \mathrm{msec}$ for stimuli with high and low primary associates, respectively) [quasi- $F(1,1)=$ $\left.8.2, \mathrm{p}>.05, \mathrm{MS}_{\mathrm{e}}=684\right]$.

RECALL task. In addition to systematic variation in RT among words within a cell of the design $\left[F(10,110)=2.5, \mathrm{p}<.05, \mathrm{MS}_{\mathrm{e}}=104,155\right]$, PAs whose stimuli have low-strength primary associates were retrieved significantly faster (mean RT $=750.3 \mathrm{msec}$ ) than PAs whose stimuli have high-strength primary 
1ssociates (mean RT $=870.9 \mathrm{msec}$ ) [quasi-F $(1,14)=$ $\left.7.7, \mathrm{p}<.01, \mathrm{MS}_{\mathrm{e}}=485,413\right]$. Also, $\mathrm{RT}$ decreased with practice [quasi- $F(8,78)=7.3, p<.01, \mathrm{MS}_{\mathrm{e}}=107,702$ ], sut this decline in RT was the same for both types of stimuli [quasi-F $(8,51)<1.0, \mathrm{MS}_{\mathrm{e}}=43,434$ ] .

Finally, while the slope of the lag function was somewhat larger for stimuli with higher strength primary associates (mean slope $=2.2$ and 1.3 for high- and lowstrength stimuli, respectively), this result was not reliable $\left[\mathrm{F}(1,11)=3.3, \mathrm{p}>.05, \mathrm{MS}_{\mathrm{e}}=12\right]$.

\section{Discussion}

Experiment II clearly demonstrates that RECALL RT is longer to stimuli with high-strength primary associates than to those with low-strength primary associates. This result is opposite to the effect observed by Shapiro (Note 4); however, as previously indicated, Shapiro's results may be due to effects of stimulus $f$ and S-R associative strength, rather than type of primary. Other procedural differences which may be relevant are that (1) Shapiro used nonprimary associates as responses, whereas in our experiment nonassociates were used; (2) Shapiro's error rate was more than $6 \%$, as compared to our error rate of less than $1 \%$; and (3) Shapiro assigned RTs of $2,270 \mathrm{msec}$ to error trials, whereas error trials were eliminated from our analyses.

\section{GENERAL DISCUSSION}

It is clear that both error rates and time to recall a memorized response to a stimulus are higher for stimulus words which have higher $f$ of English language usage. The effects of $\mathrm{m}$ are less clear, but Saltz (1967) has found that error rates are lower when the stimulus has more English language associates, and both error rate and RT trends in our Experiment I are consistent with his findings. In addition, slopes of RT lag functions were significantly steeper for high-f and low-m stimuli. Finally, the memorized response is produced more slowly to stimuli with high primary associates, and the lag function for such stimuli is somewhat, although not significantly, higher than for stimuli with low primary associates. The problem at hand is to account for this pattern of results.

Before considering the class of retrieval models referred to in the introduction, we should note why the results cannot be accounted for by encoding processes. Certainly, common and rare words differ in ways that affect their speed of perception, as Landauer and Streeter (1973) have noted. However, such effects of word $f$ are uniformly in a direction opposite to that observed in our recall items. In a preliminary study (Perlmutter et al., Note 2), we found time to name high-f words to be significantly less than time to name low-f words. In addition, Forster and Chambers (1973) obtained the same result and found that it also held for lexical decision times. Furthermore, this encoding hypothesis fails to account for the effects of our variables upon accuracy scores obtained by both Saltz (1967) and ourselves. In the absence of any evidence that our variables affect encoding or response execution in a manner which accounts for error rates and recall times, we are persuaded that it is retrieval of the associate that is affected.

An alternative explanation is that effects upon retrieval time may solely reflect differences in episodic representations under different experimental conditions. According to this line of reasoning, our differences in RT merely reflect differences in initial level of learning. Although we have provided a mathematical model along these lines which fits other data quite well (Perlmutter, Sorce, \& Myers, 1976), we do not find the argument persuasive given the present data. First, it begs the question. Why should high-m and low-f stimuli produce better learning? Second, this position would lead us to expect more pronounced lag effects with low- than with high-f stimuli, which is exactly opposite to the observed effect. This prediction follows because, as lag increases, RT for both types of stimuli would rise to a common asymptote; then the increase in RT with increases in lag should be most pronounced when the initial RT is lowest, that is, with low-f stimuli. Similarly, as practice proceeds, strengths of initially weaker associations should approach those of initially stronger associations, and, consequently, $f$ effects upon RT should decrease with practice. However, there is no evidence of an $f$ by Blocks interaction in our data.

Assuming that the observed effects have their locus in retrieval, the data appear to be most consistent with the view that semantic associates interfere with retrieval of episodically learned responses. When the stimulus is presented, its associates are searched; those which were not encoded within the context of the particular episode are rejected. Nevertheless, such preexperimental associations will influence error rates and RT. The likelihood of finding the correct associate, and the time to do so, will depend not only upon the strength of that association, but also upon the distribution of strengths of competing associates. That retrieval time is a function of strength-a conclusion consistent with repeated findings of associative strength, practice, and lag effects (Perlmutter et al., 1976)-rules out that subset of competitive search models in which search rates are independent of strengths. Other plausible search models can be rejected, but there is at least one model, with both a serial and a parallel interpretation, which provides a reasonable account of our results.

Before considering alternative search models, the following assumptions should be restated. First, the sum of strengths of associations to any of our stimuli is indexed by its $f$ of English language usage. Second, the distribution of strengths of associations to a stimulus varies as a function of stimulus $m$ and strength of the primary associate.

We recognize that unspecified correlates of our variables may play a role, and that other identifications 
with these variables are possible. However, to the extent that our data are explained by a particular model, both that model and the assumptions just summarized gain credence. Within this context, the data rule out an exhaustive search (serial or parallel) of the entire set of associations to a stimulus. Since, at a given level of $\mathrm{m}$, the average strength of relations increases with increases in stimulus $f$, the variable search rate assump. tion leads to the incorrect prediction that retrieval time will be shorter for high-f stimuli.

Two equivalent self-terminating models provide a better account of the $f$ effect. In the serial version, associates are sampled one at a time with replacement. If probability of tracing a path is equal to the proportion of total strength associated with that path, on the average the correct response will be retrieved later, as strength associated with irrelevant paths is increased. Furthermore, increasing associative strength of the memorized response will increase its retrieval probability, resulting in finding it earlier. An equivalent parallel model (Townsend, 1971, 1972) assumes that processing capacity is limited and distributed over paths as some monotonic function of associative strength, and that time to search a path is exponentially distributed with rate equal to the processing capacity allocated to that path. As stimulus $f$ increases, so does the share of the fixed capacity assigned to irrelevant paths; thus, retrieval of the memorized response is slower. Conversely, if associative strength of the memorized response is increased, the correct path receives a greater share of processing capacity, and recall will be faster. The problem with both of these self-terminating models is that average time to retrieve the correct association depends solely on its proportion of total strength. This leaves no way of accounting for the marginal effect of $m$ observed in Experiment $I$ and Saltz's experiments, nor the clear effect of strength of the primary associate obtained in Experiment II.

Such distributional phenomena might be accounted for by a modified version of the models just considered. The assumption that time to trace an associative path depends upon its proportion of total strength is maintained. However, we now assume that, when an associate is rejected, it no longer competes with the to-be-recalled associate. In a serial model (Shapiro, 1968), this can be accomplished by sampling without replacement. An analogous process in a parallel self-terminating model would be the redistribution of processing capacity among those associates which have not yet been checked (Atkinson, Holmgren, \& Juola, 1969, have considered the special case in which capacity is equally distributed over all elements to be searched). In both serial and parallel cases, the relative strength of the correct association increases faster as strength of the primary associate increases. This is true because the primary associate has a higher probability of being rejected early, and its rejection leads to larger increases in the relative strength for the correct associate when the stimulus has a highstrength primary than a low-strength primary associate. Therefore, this argument leads to the incorrect prediction of faster RT for high-strength primary stimuli, and indicates that these self-terminating models are also rejectable.

Another modification of the self-terminating models fares better. We again assume that proportion of strength accruing to the memorized association determines the speed of correct recall. The difference is that we now assume that the search set is limited to those associations which exceed some threshold of strength. Whether search is serial self-terminating with replacement or parallel self-terminating with fixed capacity, predictions are the same. We assume that with $\mathrm{m}$ constant, increased stimulus $f$ results in more competing strength above the cutoff; with $f$ held constant, increasing $m$ results in less strength per association (see Figure 1) and, therefore, fewer associations above threshold. The greater the competing strength within the search set (that is, under higher $f$ and lower $\mathrm{m}$ ), the lower the likelihood that the memorized association will be retrieved prior to some deadline and the longer RT will be if it is retrieved.

Predictions for the effects of strength of the primary associate, observed in Experiment II, are less clear under this class of models. However, longer RT in the high primary condition are predicted if we assume that total strength above the threshold is greater for high primary stimuli. The status of such an assumption is tenuous at best, but consideration of normative data (Postman, 1970) does offer some encouragement. According to these norms, our low primary stimuli typically have many relatively idiosyncratic, and therefore presumably weak, associates. On the other hand, our high primary stimuli typically have a few frequently given, and therefore presumably strong, associates. It is, therefore, reasonable that, for any specific strength threshold, the total strength of associations surpassing that threshold would be greater in the high primary cases.

To summarize, observed effects of practice, associative strength, and stimulus $f$ and $m$ can be accounted for by self-terminating models in which (1) a search set is defined on the basis of some threshold of associative strength and (2) the rate of processing an association is a function of its strength relative to that of other associations within the search set. Under the additional assumption that (3) the sum of strengths above this threshold is greater for stimuli with high- as opposed to low-strength primary associates, the observed effect of this manipulation can also be accounted for by these models.

The ratio-of-strength models are also compatible with the consistent pattern of lag effects obtained in the two experiments. In accounting for mean RT data, we have argued that the ratio of strength of the correct associate to total strength within the search set is greater under low than under high $f$, under high than under low 
$\mathrm{m}$, and under low than under the high primary associate condition. Within each pair of conditions, the one with the higher strength ratio has consistently resulted in the smaller lag effect. This follows if we recognize that lag results in a decay of strength of the correct association. Such a decrement in both numerator and denominator of the strength ratio will result in a smaller decrease of larger ratios.

Our proposed ratio-of-strength model is similar to a model proposed by Shiffrin (1970). He assumed a random serial search through a subset of informational units ("images"); the probability of the correct image being recovered on a particular draw is its proportion of the total strength in the search set. The major difference between Shiffrin's approach and our own is in our assumption that the strength of the informational units determines whether or not they are included in the search set. Shiffrin places greater emphasis on the role of the experimental task. In any event, Shiffrin's success in fitting probability correct data from a number of conditions in both free recall and paired associate experiments provides further support for the class of models we propose.

Anderson and Clayton (Note 1) also have arrived at a similar model. Specifically, they have argued that the search is parallel and self-terminating, and that capacity is limited and distributed in proportion to the strength of relationships. Their procedure complements ours nicely; while we found that time to recall an experimentally learned associate increased as a function of the strength of preexperimental associations, they found that time to recognize a preexperimental proposition (that is, some fact known prior to the experiment) increased as a function of the number of additional propositions about the subject learned in the experiment.

One other implication of the lag data is of interest. The effect of $m$ upon lag is in the opposite direction of an effect predicted by models which assume a serial scan of a reorderable list of associates (cf. Anderson \& Bower, 1973). As can be seen in Figure 2, RT is essentially the same for high- and low-m stimuli at lag zero. In terms of a buffer model, then, we can assume that the correct response is in the same position of the memory stack of high- and low-m stimuli at lag zero; as lag increases, the correct response moves down the memory stack, and RT increases. However, position on the stack, and hence RT, should asymptote faster for low- than high-m stimuli, since they presumably have shorter memory stacks. This implies that high-m stimuli should have steeper lag functions, which is the opposite of the result observed in Experiment $I$.

In conclusion, the data, together with those obtained by Saltz (1967) and Modigliani and Saltz (1969), seem most parsimoniously explained by the hypothesis that semantic knowledge plays a role in retrieval of episodic knowledge. This does not appear consonant with a fundamental assumption of episodic theory "that each event is encoded as a unique trace which is retained independently of any permanent knowledge used in setting up the trace" (Watkins \& Tulving, 1975, p. 7). Furthermore, the data place constraints on the nature of the search model, providing a basis for the rejection of several alternatives. Assuming that our semantic variables determine the proportion of total associative strength above some cutoff, two search models can be constructed which provide an account of our results.

\section{REFERENCE NOTES}

1. Anderson, J. R., \& Clayton, L. Interference with real world knowledge. Paper presented at Psychonomic Society meetings, Boston, November 1974.

2. Perlmutter, J., Harsip, J., \& Myers, J. L. The role of semantic memory in retrieval from episodic memory: Implications for a model of retrieval. In Studies of retrieval from long-term memory. Amherst: Cognitive Processes Laboratory, University of Massachusetts, 1974. Report 74-2.

3. Perlmutter, J., Sorce, P., Harsip, J., \& Myers, J. L. Further consideration of a two-trace strength model of retrieval: The effect of interpolated activity in recall. In Studies of retrieval from long-term memory. Amherst: Cognitive Processes Laboratory, University of Massachusetts, 1974. Report 74-2.

4. Shapiro, S. I. Response latencies in paired-associate learning as a function of associative strength, hierarchy. directionality, and mediation. Unpublished doctoral dissertation, Pennsylvania State University, 1966.

\section{REFERENCES}

ANDERSON, J. R., \& BowER, G. H. Human associative memory. Washington, D.C: Winston \& Sons, 1973.

Atkinson, R. C., Holmgren, J. E., \& Juola, J. F. Ptocessing time as influenced by the number of elements in a visual display. Perception \& Psychophysics. 1969, 6, 321-326.

Forster, K. I., \& ChAmbers, S. M. Lexical access and naming time. Journal of Verbal Leaming and Verbal Behavior. 1973, 12, 627-635.

Landauer, T. K., \& STreeter, L. A. Structural differences between common and rare words: Failures of equivalence assumptions for theories of word recognition. Journal of Verbal Learning and Verbal Behavior, 1973, 12, 119-131.

Modigliani. V., \& Saltz, E. Evaluation of a model relating Thorndike-Lorge frequency and $m$ to learning. Journal of Experimental Psychology, 1969, 82, 584-586.

MYERS, J. L. Fundamentals of experimental design. (2nd ed.) Boston: Allyn and Bacon, 1972.

Palermo, D. S., \& Jenkins, J. J. Word association norms. Minneapolis: University of Minnesota Press, 1964.

Paivio, A., Yuille, J. C., \& Madigan, S. A. Concreteness, images, and meaningfulness values for 925 nouns. Journal of Experimental Psychology Monograph Supplement, $1968,76,1.25$.

Perlmutter, J., Sorce, P., \& Myers, J. L. Retrieval processes in recall. Cognitive Psychology, 1976, 8, 32-63.

Postman, L. The California norms: Association as a function of word frequency. In L. Postman \& G. Keppel (Eds.), Norms of word association. New York: Academic Press, 1970:

Postman, L.. \& Underwood, B. J. Critical issues in interference theory. Memory \& Cognition, 1973, 1, 19-40.

Reder, L. M., Anderson, J. R., \& Biork, R. A. A semantic interpretation of encoding specificity. Journal of Experimental Psychology, 1974, 102, 648-656. 
Rundus, D. Negative effects of using list items as recall cues. Journal of Verhal Liarning and Verbal Behavior. 197.3. $12,4.3-50$.

Saltz. E. Thorndike-Lorge frequency and $m$ of stimuli as separatc factors in paired associate learning. Journal of Experimental Psychology. 1967, 73, 47.3-478.

ShapiRo, S. 1. Paired-associate response latencies as a function of tree association strength. Joumal of Experimentul Psychology. 1968, 77, 22.3-231.

Shiffrin. P. M. Memory Search. In D. A. Norman (Ed.), Models of human me'moñ'. New York: Academic Press, 1970.

Sternafrg. S. Memory scanning: Mental processes revealed by reaction-time experiments. American Scientist. 1969. 57. 421-457.

Theios. J. Reaction time measurements in the study of memory processes: Theory and datit. In: G. H. Bower (F.d.). The prectologe of learning and motivation (Vol. VII). New York: Academic Press, 197,3.
Thorndike, E. L.. \& Lorgif. 1. The leachers word book of 30.010) words. New York: Bureau of Publications. Teachers college, Columbia University. 1944.

ToWNSEND. J. T. A note on the identitiability of parallet and serial processes. Pircoption \& Psychophysics, 1971. 10 161-16.3.

Townsend, J. T. Some results concerning the identifiability of parallel and serial processes. British Journal of Mathematica and Statistical Pswhology'. 1972. 25. 168-199.

Watksns. M. J., \& Trisine; F. Episodic memory: Wher recognition fails. Joumal of Experimental Psychology: Gineral, 1975, 104, 5-29.

(Received for publication July 9.1975: revision accepted O(tober 27.1975 .)

\section{ERRATUM}

Griffith, D. The attentional demands of mnemonic control processes. Memory \& Cognition, 1976, 4 (IA), 103-108. The decimal points were placed incorrectly in three of the $\mathrm{MS}_{\mathrm{e}}$ erms. The corrected values are as follows:

Recall accuracy: $\mathrm{MS}_{\mathrm{c}}=9.80$

EPC during initial list processing: $\mathrm{MS}_{\mathrm{c}}=13,955.12$

EPC during recall: $\mathrm{MS}_{\mathrm{c}}=65.533 .76$

All other reported values remain unchanged. 